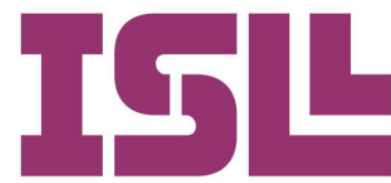

Número 7.

Enero de 2017

\section{La competencia lectora. Una aproximación teórica y práctica para su evaluación en el aula}

\author{
Reading Literacy. A Theorical and Practical \\ Approach for its Assessment in the Classroom
}

\author{
Antonio Díez Mediavilla \\ Vicente Clemente Egío \\ Universidad de Alicante
}

Pág. 22 a la 35

\section{Keywords}

Reading literacy, reading, reading comprehension, PISA, reading capacity.

\section{Abstract:}

The current investigation shows, in the first place, an analysis and theoretical approach for the concept of Reading competence. This reserach works as a basis to justify that PISA (creator of this concept) doesn't evaluate what it says to evaluate. So, we propose a new model of evaluation to assess the level of Reading comprehension, getting closer to the concept of Reading competence than Pisa has traditionally done. This research has been carried out with the participation of up to 7 classes of $4^{\circ} \mathrm{ESO}$, the realization of two teststhe before mentioned one and a second one corresponding to an excerpt of a PISA test- and the later analysis and statistical comparison between them to find differences (if any) that confirm our statements and theoretical principles. For this purpose, test $\mathrm{T}$ has been used for related samples. The statistical analysis let us check that the new Reading approach proposed in the new model of evaluation, through which we intend to approach the concept of Reading competence, had as a main consequence a better performance of the students in the realization of this test; in comparison with the PISA excerpt that was used. This better efficiency in the carrying out, by the students participating, of the new test, include many investigative implications towards the understanding of different concepts related to Reading that, in many occasions, are easily confused, as well as implications of a practical nature, providing many orientations towards educational interventions in the Reading skill area. 


\section{Introducción}

\section{La competencia lectora en PISA}

En el marco que nos ocupa se estudia el tratamiento que realiza PISA de la lectura y su evaluación. El informe del proyecto DeSeCo (Definición y Selección de Competencias clave) supone la base teórica para la entidad de la OCDE y, por tanto, una pieza fundamental en la justificación de la evaluación de lo que PISA denomina "competencia lectora", tratándose esta de una subcompetencia específica enmarcada dentro de una mayor: la competencia comunicativa. Se afirma en el proyecto DeSeCo que cada competencia clave debe "contribuir a resultados valiosos para sociedades e individuos; ayudar a los individuos a enfrentar importantes demandas en una amplia variedad de contextos; y ser relevante tanto para los especialistas como para todos los individuos" (OCDE, 2005, p.3).

El término "competencia lectora", en consonancia a las finalidades que persigue cada competencia clave viene definido, según PISA en su actualización más reciente en el ámbito de la lectura (2015), de la siguiente manera: "competencia lectora es comprender, utilizar, reflexionar y comprometerse con textos escritos para alcanzar los propios objetivos, desarrollar el conocimiento y potencial personales y participar en la sociedad" (OCDE, 2015, p.9). Una definición bastante ambiciosa, al abarcar cuestiones muy diversas y complejas; desde la aceptación del hecho de que la lectura integra aspectos cognitivos complejos como son la comprensión y reflexión a partir de textos escritos (no sólo descodificación del código escrito), hasta la admisión de que el acto lector se mueve por el logro de finalidades u objetivos, de aspecto personal y/o social.

PISA describe así una nueva realidad, de forma totalmente acertada, que se conceptualiza como un saber hacer aplicado a contextos académicos, sociales y profesionales. Se asocia entonces a habilidades prácticas que permiten al individuo desenvolverse con eficacia en la sociedad en que vivimos.

Conceptos demasiado próximos: capacidad lectora, comprensión lectora y competencia lectora

En relación al uso de los términos comprensión lectora y competencia lectora, Jiménez (2014) señala la no existencia de consenso en cuanto a su correcta utilización y advierte de la alternancia temporal en el uso de estos. En este ámbito podríamos añadir otro concepto que se une a tal confusión terminológica: la capacidad lectora.

Mediante la revisión de lo expuesto por autores expertos en la materia, podemos arrojar un poco de luz ante la oscuridad que plantea la confusión entre estos términos. Díez y García (2015) conciben el concepto de capacidad lectora como aquella "manifestación individualizada de la habilidad para descodificar, identificar la información y reflexionar sobre dicha información para poder valorarla en función de los 
saberes previos del lector y de los intereses o finalidades de la lectura" (Díez y García, 2015, p.5).

Por otro lado, Pérez-Zorrilla (2005) en relación al concepto de comprensión lectora, afirma que este supone "un proceso a través del cual el lector elabora un significado en su interacción con el texto. La comprensión a la que el lector llega se deriva de sus experiencias previas acumuladas, experiencias que entran en juego, se unen y complementan a medida que descodifica palabras, frases, párrafos e ideas del autor" (Pérez-Zorrilla, 2005, p.123).

Se trata de dos definiciones adecuadas para dos términos que, como comprobamos, son sinónimos y describen una misma noción. No es posible discernir una línea fronteriza que separe ambos conceptos, que, por tanto, podemos considerar equivalentes. Capacidad/comprensión lectora evidencian una misma realidad, referencian un concepto de lectura como construcción de puentes entre los conocimientos propios del individuo y lo nuevo que aporta el texto en el acto de lectura.

La problemática estriba, principalmente, en la confusión de cualquiera de los dos términos sinónimos expuestos con el de "competencia lectora". La competencia lectora se mueve hacia el ámbito de la pragmática, a “leer para”. Como defiende Solé (2012), así entendido el término, se relaciona con un proyecto personal que implica desarrollo, crecimiento e inserción social. Dota de un sentido totalmente práctico a la lectura; aplicar la competencia lectora supone leer, bajo un contexto específico, un determinado texto, para así poder solventar con éxito una problemática concreta que atañe al lector.

PISA aborda la problemática de creación de un modelo de evaluación de la competencia lectora ateniéndose a tres dimensiones que suponen el núcleo principal de su actuación en este campo: aspectos que se tratan, textos que se leen y situaciones en donde se establece el acto lector.

La primera dimensión, denominada aspectos, hace referencia a "estrategias mentales, enfoques o propósitos que los lectores utilizan para acercarse a los textos" (Ministerio, 2010; 34). PISA resume tales estrategias en tres grandes categorías: obtención de información, interpretación de textos y reflexión y valoración. Como afirma Saulés (2012), la comprensión total de un texto involucraría el dominio de las anteriores actividades.

La segunda dimensión, textos. De acuerdo a la actualización de 2009 (no ha sufrido ninguna modificación tal dimensión desde entonces), son organizados ajustándose a una clasificación según cuatro características: “medio (impreso y digital); ambiente (de autor y basado en el mensaje); formato (continuo, discontinuo, mixto y múltiple); tipo 
(descripción, narración, exposición, argumentación, instrucción y transacción)" (Saulés, 2012, p.48).

Tercera y última dimensión: situaciones. La situación "puede entenderse como una clasificación de tareas de lectura basadas en el uso para el que están destinados los textos, en las relaciones con los otros, que se presentan implícitas o explícitas en la tarea y en el contenido en general" (OCDE, 1999, p.23). Distingue diversos tipos de lectura, no aislados unos de otros, ya que, en no pocas ocasiones, se relacionan: lectura personal (1), pública (2), profesional (3) y educacional (4).

Para visualizar a nivel práctico el funcionamiento de PISA, respecto a la evaluación de la competencia lectora, a continuación, mostramos un pequeño fragmento de una de sus pruebas de lectura:

\section{“ALERTA DE ALERGIA A LOS CACAHUETES. \\ GALLETAS RELLENAS DE CHOCOLATE.}

Fecha de la alerta: 4 de febrero

Nombre del fabricante: Fine Foods Ltd.

Información sobre el producto: Galletas rellenas de chocolate, $125 \mathrm{~g}$

(Consumir preferentemente antes del: 18 de junio y consumir preferentemente antes del: 1 de julio).

Detalles: algunas galletas de estos lotes pueden contener trazas de cacahuete, que no están incluidas en la lista de ingredientes. Las personas alérgicas a los cacahuetes no deben comer estas galletas.

Actuación por parte del consumidor: si ha comprado estas galletas puede llevarlas al lugar donde las adquirió para que le devuelvan el dinero, o llamar al 900323334 para más información

\section{PREGUNTA 1:}

\section{¿Cómo se llama la empresa que elaboró las galletas?}

Características del marco:

- Situación: pública

- Aspecto: acceder y obtener información

Adaptado de MECD, 2013, p. 85-86

Tras esta imagen práctica sobre la evaluación que plantea PISA en el campo de la lectura, estamos en condiciones de afirmar que PISA no evalúa lo que dice evaluar; es 
decir, no valora la competencia lectora, entendida esta, en consonancia a la propia definición que propone la OCDE.

La propuesta de PISA se limita, tal como afirma Alcaraz et alt. (2013), a evaluar procesos puramente cognitivos relativos a la obtención y recuperación de información, interpretación y valoración de esta, reflexión, entre otros. Utiliza textos adecuados que responden a una diversa tipología y a diferentes situaciones de lectura. No obstante, al trabajar con estos se "olvida" del apartado pragmático y de la dimensión personal/afectiva y social que se relaciona a los fines de la lectura (aspectos clave de la competencia lectora); la finalidad que mueve la lectura en la realización de sus pruebas no es otra que la de contestar preguntas sobre la información contenida en el propio texto.

PISA utiliza el concepto de "competencia lectora" y no "leer" para, como afirma en su propio proyecto de marco conceptual más reciente (PISA 2015: DRAFT READING LITERACY FRAMEWORK), alejarse de una posible visión reduccionista de este último, que pudiera ser interpretado equivocadamente y limitado a la traducción del código escrito o a leer en voz alta. Con la finalidad de valorar algo más profundo y de acuerdo con su base teórica (DeSeCo), surge el concepto de competencia lectora ya descrito anteriormente. Sin embargo, sus pruebas no se centran en dicho concepto tan bien planteado a nivel teórico; en parte, porque la competencia lectora como tal, no resulta fácil de evaluar en el aula; en esta, el estudiante no suele tener necesidades reales de realizar lecturas pragmáticas para así solventar problemáticas sociales y personales (como pudiera ser la lectura de un horario de trenes para escoger el que más le convenga; o la lectura de una determinada oferta de trabajo que pudiera interesarle, entre muchos otros ejemplos).

Mientras que la valoración de la comprensión lectora e incluso su mejora mediante estrategias como el aumento de vocabulario y realización de resúmenes, tal como señalan Ripoll y Aguado (2014), o la importancia de la lectura dialógica que expone Gutiérrez Fresneda (2016)- se encuentran en un estado bastante avanzado; completamente distinto es el caso que nos ocupa, concerniente a la competencia lectora.

Se concreta nuestra actuación, primero en el tratamiento de dos de los aspectos ya citados que trabaja PISA: integrar e interpretar (aspecto 2) reflexión y valoración (aspecto 3). Este último, por la profundización en el ámbito de relación e interacción entre texto y lector que comprende, es el más cercano a la denominada competencia lectora y, por tanto, el que más nos podría interesar en este estudio. Creemos, no obstante, que si trabajamos con los dos aspectos señalados podríamos obtener unos resultados más interesantes al fusionar aspectos relacionados con la capacidad lectora (aspecto 2) y con la participación activa, el diálogo lector/texto, del aspecto 3. 
Ambas pruebas compartirán, además de los aspectos trabajados, textos y cuestiones de iguales características (género del texto, formato, situación, entre otros).

Los objetivos de nuestra investigación se centrarán pues en: a) Elaborar un instrumento de comprensión lectora más próximo al concepto de competencia lectora que aquellos elaborados por PISA y b) Analizar las relaciones de aproximación o distanciamiento en los resultados de eficacia lectora del alumnado ante dos pruebas equivalentes: una parte de una prueba de PISA y otra de elaboración propia.

Estos objetivos se relacionan con una hipótesis de trabajo según la cual el alumnado respondería de manera más significativa y eficaz a la nueva propuesta. Esta hipótesis se explica mediante la fundamentación principal de que la nueva prueba se crea incorporando al proceso una necesidad de lectura, descrita en el marco de lectura pragmática de utilización directa (previo a la lectura del texto en cuestión), con la ambición de otorgar una mayor significatividad y finalidad a la lectura; lo cual va a favorecer directamente la consecución de una participación más comprometida del alumnado, que habrá de involucrarse en la lectura a realizar para así poder resolver la problemática descrita.

\section{Método}

Los participantes en este estudio conforman un amplio grupo de 141 estudiantes pertenecientes a un total de 7 grupos de $4^{\circ}$ de ESO distribuidos en 4 institutos enmarcados dentro de la comarca de la Vega Baja (Alicante).

En cuanto a los instrumentos. Debido a la gran extensión de estos, no se encuentran incluidos en el presente artículo. De manera que, a continuación, citaremos las características principales de ambas pruebas.

\section{Parte de una prueba PISA.}

Texto: Destino Buenos Aires. Antoine de Saint-Exupéry. Vol de Nuit.

- Cuestiones de lectura:

1. ¿Cómo se siente Rivière en su trabajo? Utiliza el texto para justificar tu respuesta.

2. Destino Buenos Aires se escribió en 1931. ¿Crees que hoy en día las preocupaciones de Rivière serían parecidas? Justifica tu respuesta."

(Adaptado de Ministerio, 2013, p.90)

2. Prueba de elaboración personal propia.

La finalidad lectora. Marco de lectura pragmática.

Estás hablando con dos amigos, Alejandro y Daniel, y te explican el siguiente suceso: El viernes pasado, tras ver una película, Alejandro acabó llorando de emoción. Mientras, Daniel no entendía cómo era posible que llorara Alejandro, ya que defiende que las películas son solo eso; películas, nada más que historias fingidas. Tus amigos te piden tu opinión al respecto. Por si te sirve para reflexionar sobre la cuestión que te plantean tus amigos, te proponemos que leas el siguiente texto 
Texto: Macondo. Gabriel García Márquez. Cien años de soledad.

- Cuestiones de lectura:

1. ¿Por qué se sintió estafada la gente de Macondo tras ver la película?

2. Tanto en la situación de Alejandro como en la de la gente de Macondo, se refleja una implicación sentimental a la hora de ver películas. La pregunta que Daniel te hace es la siguiente: “¿es ilógico que una persona llore al ver una película?” Justifica tu respuesta.

Esta segunda propuesta sigue todos los parámetros de PISA, incluyendo un único aspecto novedoso: el marco de lectura pragmática. Este conforma la clave para el trabajo de la competencia lectora. Consiste en un pequeño fragmento de texto que no cuenta con la pretensión de suponer un objeto de estudio en sí, sino de contextualizar la lectura y situar al alumno/a para su uso práctico; primero se le pone en situación, y más adelante aparece la lectura en cuestión (texto: Macondo), la cual tiene la finalidad de constituir una valiosa fuente de información válida como soporte para resolver la problemática descrita en tal marco.

En relación a las pruebas y su realización por el alumnado, así como su corrección, cabe realizar varias indicaciones.

Un total de 7 grupos (pertenecientes a 4 institutos públicos) realizaron las pruebas, utilizando un tiempo máximo de 50 minutos (en ninguna clase se necesitó agotar todo este tiempo), ocupando de tal manera parte de una sesión del área de Lengua y Literatura. No se les aportó información relativa a la procedencia de las pruebas. Fueron realizadas en un espacio de tiempo corto comprendido entre 4-15 de mayo.

A la hora de valorar las pruebas, estas fueron calificadas de acuerdo al grado de cumplimiento de las expectativas de respuestas descritas en el anterior apartado. De acuerdo con PISA, la pregunta 1 se valoró con una puntuación de 0-1-2, mientras que en la pregunta 2 se hizo lo propio con 0-2 de acuerdo al éxito en la respuesta.

Una vez valoradas las pruebas, se avanzó en el estudio mediante la realización del análisis estadístico. Para ello, se llevó a cabo un contraste de hipótesis mediante la comparación pregunta a pregunta de las pruebas A (parte de prueba PISA) y B (prueba de elaboración personal propia). Introducidos los datos en el SPSS, se observaron las frecuencias y estadísticos descriptivos relacionados a las respuestas a las preguntas; para después continuar con un análisis detallado mediante la prueba $\mathrm{T}$ de Student para muestras relacionadas, mediante la cual se comprobó el rendimiento de una misma población (141 estudiantes) en dos distintas variables (prueba A y prueba B) con la finalidad de corroborar o refutar la hipótesis nula (no existencia de diferencias entre los resultados de una y otra prueba). De manera que se realizó el mismo análisis en dos ocasiones: una para la pregunta 1 y otra para la pregunta 2.

ISL, vol. 7, 2017, págs. 22-35 ISSN: $2340-8685$
Díez Mediavilla, A. y Egío Clemente, V. (2017): La competencia lectora. Una aproximación teórica y práctica para su evaluación, Investigaciones Sobre Lectura, 7, 22-35. 
No se comparan los resultados globales de los alumnos/as en las pruebas debido a que la clave del estudio se encuentra en conocer cómo influye el planteamiento de la nueva prueba elaborada para cada tipo de pregunta y su correspondiente aspecto trabajado.

Tras realizar el pertinente análisis estadístico, presentamos los resultados obtenidos en nuestro estudio. En las tablas que mostramos a continuación utilizaremos la letra "A" para hacer referencia a la parte de la prueba extraída de PISA, mientras que la letra "B" identifica la nueva prueba de lectura. En primer lugar, comenzaremos con el análisis relacionado a la pregunta 1 (aspecto: integrar e interpretar).

\begin{tabular}{r|c|c|c|c} 
& $\mathrm{N}$ & Media & Desv. típ. & Varianza \\
\hline Respuestas 1 A & 141 & 1,39 &, 817 &, 668 \\
Respuestas 1 B & 141 & 1,50 &, 798 &, 637
\end{tabular}

Tabla 1. Estadísticos descriptivos pregunta 1

En la Tabla 1 podemos observar diversos estadísticos descriptivos. Nos centramos, con especial interés, en las medias obtenidas; de primeras, contemplamos una media mayor por lo que respecta a las respuestas de la prueba B frente a la prueba A $(1.50 \mathrm{y}$ 1.39 , respectivamente).

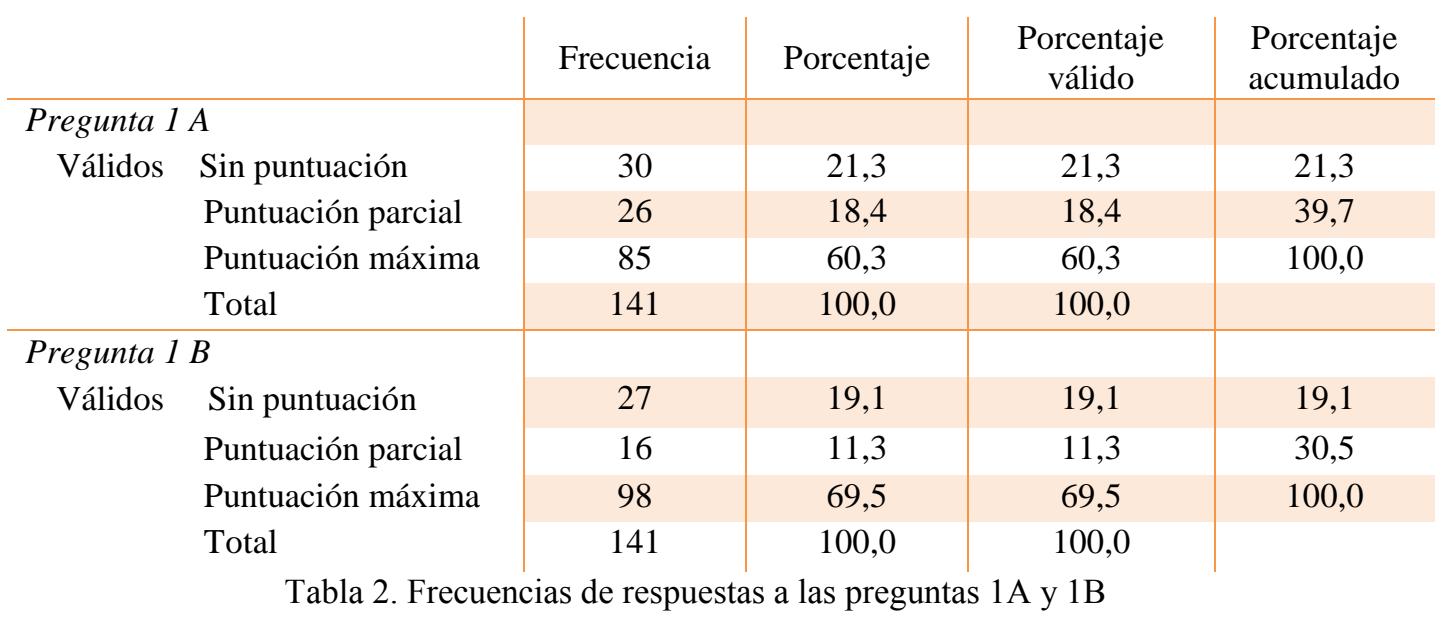

La Tabla 2 presenta las frecuencias de respuestas; mediante ellas nos adentramos en la corrección de ambas pruebas de lectura y las repeticiones de puntuaciones obtenidas en función del rendimiento en la respuesta a las preguntas 1A y 1B.

La descripción de los estadísticos de tipo descriptivo y frecuencia no permite conocer la existencia de diferencias estadísticamente significativas, por lo que procedemos al contraste de hipótesis mediante la Prueba T para muestras relacionadas. 


\begin{tabular}{|c|c|c|c|c|c|c|c|c|}
\hline & \multicolumn{8}{|c|}{ Diferencias relacionadas } \\
\hline & Media & $\begin{array}{c}\text { Desviación } \\
\text { típ. }\end{array}$ & $\begin{array}{l}\text { Error } \\
\text { típ. de la } \\
\text { media }\end{array}$ & $\begin{array}{r}95 \% \mathrm{Ir} \\
\text { confia } \\
\text { dif }\end{array}$ & $\begin{array}{l}\text { rvalo de } \\
\text { a para la } \\
\text { encia }\end{array}$ & $\mathrm{t}$ & $\mathrm{gl}$ & $\begin{array}{c}\text { Sib. } \\
\text { (Bilateral) }\end{array}$ \\
\hline & & & & Inferior & Superior & & & \\
\hline $\begin{array}{l}\text { Par 1: } \\
\text { Respuestas 1 A } \\
\text { Respuestas 1 B }\end{array}$ &,- 113 & 1,109 & 0,93 &,- 298 &, 071 & $-1,215$ & 140 & ,226 \\
\hline
\end{tabular}

La Tabla 3 muestra los datos más relevantes de nuestro análisis estadístico. La comparación del rendimiento en la contestación a las preguntas de la prueba A y prueba B nos permite corroborar la hipótesis nula: no existencia de diferencias estadísticamente significativas entre las puntuaciones de respuesta de una y otra prueba. Esto es comprobado por el hecho de que el valor de P. (Sig. Bilateral) es mayor a .05, circunstancia que no nos permite rechazar la hipótesis nula y, por tanto, advierte de la no diferencia entre los resultados de las pruebas.

Este primer análisis no apoya nuestra hipótesis de investigación. Sin embargo, como señalábamos en el marco teórico, el concepto de competencia lectora y su entendimiento práctico del acto de leer como base para resolver finalidades de lectura que responden a necesidades de participación en situaciones personales y/o sociales, se relaciona de forma más directa y clara con el aspecto 3: reflexionar y valorar. Por lo tanto, avanzamos hacia el análisis de la pregunta 2, comenzando con la presentación de los estadísticos descriptivos.

\begin{tabular}{r|c|c|c|c}
\multicolumn{2}{c|}{$\mathrm{N}$} & Media & Desv. Típ. & Varianza \\
\hline Respuestas 1 A & 141 & 1,01 & 1,004 & 1,007 \\
Respuestas 1 B & 141 & 1,63 &, 778 &, 606
\end{tabular}

Tabla 4. Estadísticos descriptivos pregunta 2

La Tabla 4 muestra los descriptivos típicos a nivel estadístico. Centrándonos en la media, observamos una diferencia teóricamente amplia entre ambas pruebas (1.01 en la prueba A, frente a 1.63 en la prueba B). A continuación, observamos las frecuencias de respuesta en las distintas pruebas.

ISL, vol. 7, 2017, págs. 22-35 ISSN: $2340-8685$
Díez Mediavilla, A. y Egío Clemente, V. (2017): La competencia lectora. Una aproximación teórica y práctica para su evaluación, Investigaciones Sobre Lectura, 7, 22-35. 


\begin{tabular}{|c|c|c|c|c|c|}
\hline & & Frecuencia & Porcentaje & $\begin{array}{l}\text { Porcentaje } \\
\text { válido }\end{array}$ & $\begin{array}{l}\text { Porcentaje } \\
\text { acumulado }\end{array}$ \\
\hline \multicolumn{6}{|c|}{ Pregunta 2 A } \\
\hline \multirow[t]{3}{*}{ Válidos } & Sin puntuación & 70 & 49,6 & 49,6 & 49,6 \\
\hline & Puntuación máxima & 71 & 50,4 & 50,4 & 100,0 \\
\hline & Total & 141 & 100,0 & 100,0 & \\
\hline \multicolumn{6}{|c|}{ Pregunta $2 B$} \\
\hline \multirow[t]{3}{*}{ Válidos } & Sin puntuación & 26 & 18,4 & 18,4 & 18,4 \\
\hline & Puntuación máxima & 115 & 81,6 & 81,6 & 100,0 \\
\hline & Total & 141 & 100,0 & 100,0 & \\
\hline
\end{tabular}

El estudio de la Tabla 5 nos permite distinguir las diferencias encontradas en las frecuencias de respuesta de ambas pruebas. Cabe destacar que, desde un primer momento, podemos observar grandes desigualdades en este punto; pues mientras en la prueba $\mathrm{A}$ poco más de la mitad (71) obtuvieron la puntuación máxima, la eficacia en la respuesta a esta pregunta en la prueba B se incrementó en gran medida (115). Asimismo, solamente 26 alumnos/as de la prueba B no obtuvieron puntuación, mientras que la prueba A obtuvo una frecuencia en la valoración "sin puntuación" de hasta 70.

A continuación, desarrollamos el análisis de la Prueba $T$ para muestras relacionadas, para así conocer la importancia o no, de tales diferencias descritas.

\begin{tabular}{c|c|c|c|c|c|c|c|}
\multicolumn{1}{c|}{} & \multicolumn{9}{c}{ Diferencias relacionadas } \\
\cline { 2 - 8 } & Media & $\begin{array}{c}\text { Desviación } \\
\text { típ. }\end{array}$ & $\begin{array}{c}\text { Error típ. } \\
\text { de la } \\
\text { media }\end{array}$ & $\begin{array}{c}95 \% \text { Intervalo de } \\
\text { confianza para la } \\
\text { diferencia } \\
\text { Inferior }\end{array}$ & T & Superior & Sib. \\
(Bilateral)
\end{tabular}

A través del estudio de la Tabla 6 establecemos la existencia de diferencias significativas no debidas al azar en la respuesta de la muestra a la pregunta 2 de la prueba B. La razón se encuentra en el hecho de que $\mathrm{P}$ (sig. Bilateral) $<.05$ por lo que, en el contraste con la hipótesis nula, se rechaza esta última. Entonces, se admite que la diferencia de medias (0.624) es significativa; el alumnado ha actuado con mayor eficacia en la resolución a la pregunta 2 de la prueba B.

Por lo tanto, las desigualdades observadas a nivel de medias (1.01 frente a 1.64), así como las frecuencias de respuestas de puntuación máxima tan favorables a la prueba $\mathrm{B}$, resultan estadísticamente significativas. 


\section{Discusión}

Nuestro primer objetivo de investigación se relaciona con la creación de una prueba de comprensión lectora más cercana a la competencia lectora; de acuerdo con la afirmación de Alcaraz et alt. (2013) por la que defiende que PISA no evalúa tal concepto.

La nueva propuesta desarrollada cumple con la finalidad de concretar la temática que trata la lectura y exportarla a una situación personal y social hipotética que involucre en mayor medida al lector en la lectura. Mediante estos aspectos innovadores, se le otorga un mayor significado a la lectura, aproximándonos al concepto de competencia lectora, entendido como "comprender, utilizar, reflexionar y comprometerse con textos escritos para alcanzar los propios objetivos, desarrollar el conocimiento y potencial personales y participar en la sociedad" (Ministerio, 2010, p. 34).

Nuestro segundo objetivo de investigación supone la comprobación de la existencia de diferencias significativas a nivel de respuesta a las dos preguntas que componen las pruebas de lectura. Objetivo que se relaciona directamente con nuestra hipótesis de trabajo, la cual afirma que existen tales diferencias y que, además, son favorables a la nueva prueba de lectura; lo que implica que el alumnado obtendrá mejores resultados en esta última. Ello se debe a que el marco pragmático de lectura ha de lograr involucrar en mayor medida al alumnado, favoreciendo así el entendimiento, motivación y realización de la tarea propuesta.

En consecuencia a los resultados obtenidos por medio de la experimentación y su posterior análisis estadístico, estamos en condiciones de afirmar que la hipótesis de investigación se ve cumplida. Sin embargo, cabe matizar la anterior afirmación; el alumnado ha realizado mejor la nueva prueba de lectura, aunque tal hecho sólo se refleja de manera relevante en una de las preguntas y aspectos trabajados (pregunta 2; reflexión y valoración). La pregunta 1 y su aspecto de integración e interpretación, a pesar de reflejar una mayor media en la prueba $\mathrm{B}$, no ha podido ser demostrado la existencia de una diferencia real en cuanto a la actuación de los estudiantes en términos de eficacia para la pregunta 1 de ambas pruebas.

Llegados a este punto, cabe recalcar la idea que la competencia lectora, como tal y entendida de acuerdo a PISA y su marco teórico, resulta muy difícil de valorar en el aula. Para ello, resultaría totalmente imprescindible la creación de necesidades reales de lectura para los estudiantes, que le sean un apoyo para resolver situaciones específicas personales y sociales. Cabe resaltar que la nueva prueba desarrollada en este estudio trata de orientarse más, y lo consigue como hemos comprobado en los resultados, al ámbito pragmático que plantea la competencia lectora. El hecho de que afirmemos que los resultados confirman que la nueva prueba se aproxima más a tal concepto viene justificado por el fundamento de que el acto de lectura en esta, al necesitar de una mayor

ISL, vol. 7, 2017, págs. 22-35 ISSN: $2340-8685$
Díez Mediavilla, A. y Egío Clemente, V. (2017): La competencia lectora. Una aproximación teórica y práctica para su evaluación, Investigaciones Sobre Lectura, 7, 22-35. 
participación del lector, consigue que este se torne protagonista en el diálogo texto-lector al tener que resolver unas necesidades de lectura que ya no son las típicas de leer para contestar unas preguntas que implican el trabajo en el plano cognitivo solamente, sino que ahora interviene la "necesidad" de nutrirse del texto para poder dar una respuesta razonada ante una problemática social y/o personal concreta.

Los resultados obtenidos en el presente estudio conllevan numerosas implicaciones al ámbito educativo, al suponer un giro importante en cuanto al trabajo en el campo de la lectura. Al trasladar la actualidad investigativa en el campo la comprensión y competencia lectora al ámbito práctico, se concreta una propuesta de trabajo en el marco de la lectura de la que los docentes de cualquier nivel (Primaria y Secundaria principalmente) pueden extraer interesantes conclusiones. Será importante así que los docentes comiencen a trabajar la lectura de acuerdo a lo expuesto; trabajando mucho, y de forma cuidadosa, el contexto prelectura y considerando finalidades lectoras que interesen al alumnado y favorezcan la realización de lecturas realmente prácticas, facilitando así, en la medida de lo posible, la aplicación de la denominada competencia lectora.

Por lo que respecta a las implicaciones investigativas. La realización de este estudio supone un avance práctico importante en el campo de estudio de la competencia lectora. Un ámbito que se encuentra en auge, pero del que todavía no hay demasiadas evidencias prácticas investigativas.

La verificación del hecho de que la prueba creada se acerca, y aproxima más al alumnado, a lo que PISA denomina competencia lectora, abre un amplio espacio sobre el que continuar la investigación. Quedan abiertas, por tanto, líneas de trabajo que abarquen la realización de pruebas de lectura completas que sigan el nuevo planteamiento expuesto. Cabría plantearse también, llegados a este punto, la consideración, en el planteamiento de las pruebas, de los distintos niveles de comprensión lectora y su relación con la competencia lectora, hecho sobre el que Jiménez (2015) apunta que, en determinado momento, el acto de comprender desemboca en una manifestación práctica (lo que entendemos por competencia lectora). Resultaría también interesante comenzar a plantearse cómo se traduce el trabajo de la competencia lectora al ámbito de la lectura digital, pues las habilidades involucradas en este tipo de lectura difieren respecto a las propias de la lectura tradicional, tal como señalan tanto Leu Donald et al. (2015) como Coiro y Dobler (2007). 


\section{Bibliografía}

Alcaraz, N., Caparrós, R. M., Soto, E., Beltrán, R., Rodríguez, A., \& Sánchez, S. (2013). ¿Evalúa PISA la competencia lectora? Revista de Educación, 577-599.

Coiro, J., \& Dobler, E. (2007). Exploring the online reading comprehension strategies used by sixth-grade skilled readers to search for and locate information on the Internet. Reading Research Quarterly, 214-257.

Díez, A., \& García-Velasco, A. (2015). La evaluación de la competencia lectora. Lenguaje y textos, 35-44.

Gutiérrez Fresneda, R. (2016). La lectura dialógica como medio para mejorar la comprensión lectora. Investigaciones Sobre Lectura, 52-58.

Jiménez, E. (2013). Comprensión lectora VS Competencia lectora: qué son y qué relación existe entre ellas. Investigaciones Sobre Lectura, 65-74.

Jiménez, E. (2015). Niveles de la comprensión y competencia lectoras. Lenguaje y textos, $19-25$.

Leu Donald, J., Elena, F., Chris, R., Cheryl, M., Clint, K., \& Nicole, T. (2015). The New Literacies of Online Research and Comprehension: Rethinking the Reading Achievement Gap. Reading Research Quarterly, 37-59.

Mendoza, A. (1998). Tú, lector Aspectos de la interacción texto-lector en el proceso de lectura. Barcelona: Octaedro.

Mendoza, A. (2001). El intertexto lector, El espacio de encuentro de las aportaciones del texto con las del lector. Cuenca: Universidad de Castilla la Mancha.

Ministerio. (2010). La lectura en PISA 2009. Marcos y pruebas de evaluación. Madrid: Omagraf.

Ministerio. (2013). Marcos y pruebas de evaluación de 2012. Matemáticas, lectura y ciencias. Madrid: INEE.

OCDE. (2000). La medida de los conocimientos y las destrezas de los alumnos. Un nuevo marco de evaluación. Madrid: INCE.

OCDE. (2005). Proyecto DESECO: La definición y selección de competencias clave. Resumen ejecutivo (versión electrónica). Obtenido de http://www.deseco.admin.ch/bfs/deseco/en/index/03/02.parsys.78532.download List.94248.DownloadFile.tmp/2005.dscexecutivesummary.sp.pdf.

OECD. (2015). PISA 2015: Draft Reading Literacy Framework. París: OECD.

Pérez Gómez, Á. (2007). La naturaleza de las competencias básicas y sus aplicaciones pedagógicas. Cantabria: Cuadernos de educación de Cantabria.

ISL, vol. 7, 2017, págs. 22-35 ISSN: $2340-8685$
Díez Mediavilla, A. y Egío Clemente, V. (2017): La competencia lectora. Una aproximación teórica y práctica para su evaluación, Investigaciones Sobre Lectura, 7, 22-35. 
Pérez-Zorrilla, M. J. (2005). Evaluación de la comprensión lectora: dificultades y limitaciones. Revista de educación, 121-138.

Ripoll, J. C., \& Aguado, G. (2014). La mejora de la comprensión lectora en español: un meta-análisis. Revista de Psicodidáctica, 27-44.

Saulés, S. (2012). La competencia lectora en PISA. Influencias, innovaciones y desarrollo. México, D.F.: INEE.

Solé, I. (2012). Competencia lectora y aprendizaje. Revista Iberoamericana de educación, 43-61. 